\section{\$1. Symmetry Structure of the Hyperbolic Bifurcation without Reflection of Periodic Orbits in the Standard Map}

Hirose, K., *Murakami, W., *Murakami, C., *Ichikawa, Y.H.

(*College of Engineering, Chubu University)

Interplay between the chaotic and the regular motion in the few degrees of Hamiltonian systems determines stochastic properties of nonlinear dynamical systems. In the series of studies of the periodic orbits and the accelerator modes 1) in the standard map

$$
\left(\begin{array}{c}
q_{n+1} \\
p_{n+1}
\end{array}\right)=\left(\begin{array}{c}
q_{n}+p_{n}+f\left(q_{n}\right) \\
p_{n}+f\left(q_{n}\right)
\end{array}\right)
$$

with the mapping function

$$
f(q)=A \sin 2 \pi q,
$$

we have explored the island structure surrounding the periodic orbits in terms of the higher order symmetry analysis. Although the onset of the unstable bifurcation is determined by the linearized tangent map around the fixed point or the periodic orbit, the completion of the bifurcation process depends on the global property of the nonlinear map. In earlier study, we have observed that the period-3 step1 accelerator mode in the standard map undergoes the hyperbolic bifurcation without reflection, and no symmetry lines pass through the newly born periodic islands.2) Now, we have found that at the larger value of the stochastic parameter $A$, the period-4 orbit undergoes the hyperbolic bifurcation without reflection, where the periodic structure can not be determined by the known symmetry properties.

The purpose of this study is to analysis in detail of the hyperbolic bifurcation without reflection structure and specify the property of their bifurcation, using for the higher order symmetry analysis method to expand the known symetry lines.

The standard map eq.(1) is decomposed into the involution with the momentum inversion as

$$
T=J_{1}(n) \circ J_{0}(m)
$$

where the involutions $J_{0}(m)$ and $J_{1}(n)$ are defined as to satisfy the conditions $J_{0}(m) \circ J_{0}(m)=1, J_{1}(n) \circ$ $J_{1}(n)=1$. The $j$-th order involution $J_{+j}(m)$ and $J_{-j}(n)$ are given as

$$
\begin{aligned}
& J_{+j}(m)=T^{j-1} J_{1}(m) \\
& J_{-j}(n)=T^{-j} J_{0}(n) .
\end{aligned}
$$

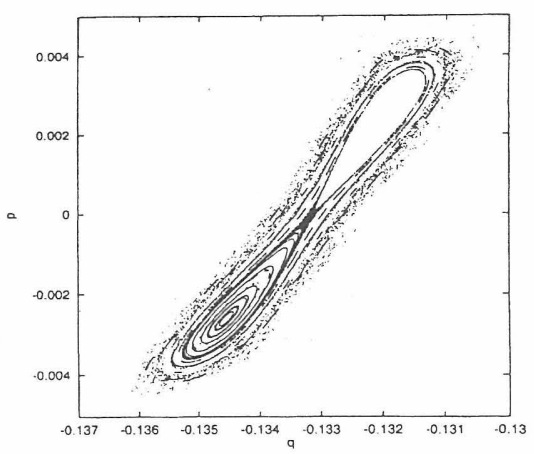

Figure 1: Hyperbolic bifurcation without reflection observed for the value of $A=0.6735$.

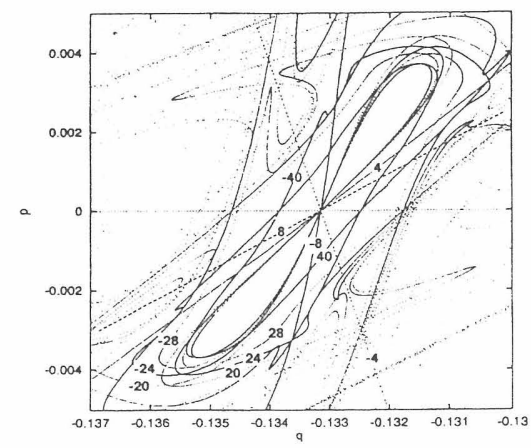

Figure 2: Higher order symmetry lines for the value of $A=0.6735$.

The fixed points of the involution $J_{j}(m)$ define a $j$-th order symmetry line $\gamma_{j}(m)$ as

$$
\gamma_{j}(m):\left\{R / J_{j}(m) R=R\right\}
$$

where $R$ stands for the two dimensional vector with the component $p$ and $q$. The intersection of $\gamma_{j}(m)$ and $\gamma_{k}(n)$ determines the periodic orbit of the standard map, $T$ of which period $N$ divides $|j-k|$. The relation

$$
T^{n} \gamma_{j}=\gamma_{j+2 n}
$$

plays the key roll in constructing the higher order symmetry lines from the fundamental symmetry lines $\gamma_{0}(m)$ and $\gamma_{-1}(n)$.

Fig.1 shows the hyperbolic bifurcation without reflection. It is clearly understand that the particles does not reflection among the separatrix. Using for the higher order symmetry line eq.(6) for Fig.1 is Fig.2. The higher order symmetry lines approach asymptotically to the separatrix at the destabilized periodic orbit.

\section{References}

1). Y.H.Ichikawa,T.Kamimura, and

T.Hatori,Physica D80,256(1987)

2). Kei.Hirose,S.Saitô,Y.Nomura, and Y.H.Ichikawa: J.Plasma and Fusion Research.75 (1999), pp.65-74 